\title{
Picroside-I attenuated isoproterenol-induced heart damage via modification of cardio-morphology, infarct size and inflammatory cascade
}

\author{
Yi Hao ${ }^{1 *}$, Zhixiong Cui ${ }^{1}$, Shuang Zhang ${ }^{2}$, Tong Liu \\ ${ }^{1}$ Department of Cardiac Surgery, ${ }^{2}$ Department of Radiology, Beijing Luhe Hospital, Capital Medical University, Beijing 101149 , \\ China \\ *For correspondence: Email: hythia@yahoo.com; Tel/Fax: +86 $01069543901-1105$
}

Sent for review: 5 March 2019

Revised accepted: 26 January 2020

\begin{abstract}
Purpose: To study the effect of picroside-I (PIC-I) on isoproterenol (ISO)-induced heart damage in rats through determination of infarct size, antioxidant enzymes, cardiac/inflammatory and apoptotic markers, as well as cardio-morphology.

Methods: A total of 32 rats were divided equally into 4 groups. Rats in normal control group were treated with saline only, while myocardial infarction (MI) rat model was prepared by intraperitoneal (i.p.) injection of ISO at a concentration of $100 \mathrm{mg} / \mathrm{kg}$. Rats pretreated with PIC-lat dose $10 \mathrm{mg} / \mathrm{kg}$ (i.p) for 28 days and administered with isoproterenol. Another group of rats was administered only with PIC-I (10 $\mathrm{mg} / \mathrm{kg}$ ) for 28 days.

Results: After 28 days of pretreatment with PIC-I, there were significant increases in arterial blood pressure and cardiac antioxidants, as well as marked decreases in infarct size, cardiac markers, inflammatory markers and apoptotic markers in rats with ISO-induced heart damage, when compared with rats given ISO alone. Rats administered PIC-I showed better histology, with reduced necrosis and prominent cardiac fibers.

Conclusion: PIC-1 pre-treatment for 28 days significantly reversed elevations in infarct size, cardiac/inflammatory and apoptotic markers, and also improved antioxidant status and cardiac morphology in rats with ISO-induced heart damage.
\end{abstract}

Keywords: Picroside-I, Isoproterenol, Infarct size, Inflammation, Apoptosis

\begin{abstract}
This is an Open Access article that uses a fund-ing model which does not charge readers or their institutions for access and distributed under the terms of the Creative Commons Attribution License (http://creativecommons.org/licenses/by/4.0) and the Budapest Open Access Initiative (http://www.budapestopenaccessinitiative.org/read), which permit unrestricted use, distribution, and reproduction in any medium, provided the original work is properly credited.

Tropical Journal of Pharmaceutical Research is indexed by Science Citation Index (SciSearch), Scopus, International Pharmaceutical Abstract, Chemical Abstracts, Embase, Index Copernicus, EBSCO, African Index Medicus, JournalSeek, Journal Citation Reports/Science Edition, Directory of Open Access Journals (DOAJ), African Journal Online, Bioline International, Open-J-Gate and Pharmacy Abstracts
\end{abstract}

\section{INTRODUCTION}

Cardiovascular diseases (CVDs), especially ischemic heart disease (IHD) are leading contributors to global mortality and morbidity. The World Health Organization (WHO) and China Heart Failure Symposium have indicated that nearly $50-60 \%$ of total CVDdeath rate are due to myocardial infarction (MI)[1].Myocardial infarction (MI) is an acute myocardial damage (necrosis/apoptosis) which is manifested in imbalance between myocardial blood delivery (supply) and its demand (requirement)[2]. The pathophysiology of $\mathrm{Ml}$ is not fully understood. However, inflammatory response, necrosis, apoptosis, oxidative stress, hyperlipidemia and 
$\mathrm{Ca}^{2+}$ overload have been identified as the major factors [3]. Isoproterenol (ISO) is a catecholamine (synthetic) used for treating bradycardia. However, administration of high dose of ISO (more than $85 \mathrm{mg} / \mathrm{kg}$ ) causes increased cardiac oxidative stress owing to autooxidation of catecholamine, followed by cascade of detrimental events like inflammation, mitochondrial dysfunction (loss of energy-ATP), apoptosis $\left(\mathrm{Ca}^{2+}\right.$ overload), necrosis and infarctlike lesion, ultimately resulting in myocardial injury/damage $[4,5]$.

Isoproterenol-induced cardiotoxicity is a well accomplished and acceptable $\mathrm{MI}$ model since it mimics the pathophysiology of human MI $[6,7]$. Moreover, it is associated with less mortality and it is a non-invasive method. The current modern synthetic anti-Ml drugs (aspirin, clopidogrel, roglycerin, atenolol, bivalirudin and heparin) are relatively effective, but result in serious adverse effects such as bradycardia, angioedema, hypotension, hepatoxicity and serious back pain, apart from being expensive [8]. Hence, there is need for development of potent natural cardioprotective agents. Natural drugs are well tolerated, cheap and free from adverse effects, when compared to synthetic drugs [9]. Several studies have demonstrated the cardioprotective efficacy of many natural phytocomponents with potent anti-apoptotic, anti-inflammatory, and antioxidant properties against ISO-induced $\mathrm{MI}$ or cardiotoxicity $[3,7]$.

Picroside-I (PIC-I) is an iridoid glycoside extracted from Picrorhiza species such as Picrorhiza scrophulariflora and Picrorhiza kurroa (rhizome and roots). Many Picrorhiza species have been popularly used in Chinese and Indian Traditional Medicine for several years for treating various illnesses $[10,11]$. The other major bioactive components of Picrorhiza species are picroside II and III (kutkin) and kutkoside [12]. Studies have demonstrated that PIC-I has several pharmaceutical properties such as antiinflammatory [13], anti-oxidant [14], antiapoptotic [15], anti-cancer [12], anti-asthma [16] as well as neuroprotective, neuritogenic [17] and hepatoprotective effects [18]. Previously, Kumar and others [19], as well as Nandave and his colleagues [20] reported that Picrorhiza kurroa which is rich in picrosides produced strong cardioprotective effect against ISO-induced cardiotoxicity. Moreover, recently, Li and his coworkers [21] demonstrated that picroside II (another major iridoid from Picrorhiza species) protected the myocardium from ISO-induced ischemic myocardial damage. Therefore, it can be reasonably hypothesized that picroside I might also display cardioprotective effect in ISO- induced MI model. Thus, the current study was designed to screen the protective efficacy of PICI against ISO induced $\mathrm{MI}$ model by assessing infarct size, antioxidant status, hemodynamic parameters, cardiac markers, inflammatory markers and cardiac morphology in rats.

\section{EXPERIMENTAL}

\section{Chemicals and reagents}

Picroside-I (PIC-1), formaldehyde, triphenyl tetrazolium chloride (TTC), isoproterenolhydrochloride (ISO-HCl), phosphate buffered saline solution (PBS) and Tween 20 were bought from Sigma-Aldrich (MA, USA). All the other solutions, reagents (chemicals) used in the present experiments were purely analytical or HPLC grade.

\section{Experimental animals}

Totally 32 healthy male SD rats weighing $250 \pm$ $10 \mathrm{~g}$ were bought and housed under temperature of $22 \pm 2{ }^{\circ} \mathrm{C}$ and $55-60 \%$ humidity in an environment with 12-h light and dark cycle (standard lab condition). All rats were free to access water and standard rat pellet (ad libitum). This animal study was approved by the ethical board of Capital Medical University, and carried out in line with the guidelines of National Institutes Health.

\section{Rat grouping}

Totally 32 healthy male SD rats was used for this study by dividing them into 4 equal groups after 10 days of acclimatization period. The control rats received only saline for 28 days, while rats in MI model group were injected (ip) with ISO at a concentration of $100 \mathrm{mg} / \mathrm{kg}$ for 2 consecutive days (on $29^{\text {th }}$ and $30^{\text {th }}$ days). Rats in another group were pretreated with PIC-I at a dose of 10 $\mathrm{mg} / \mathrm{kg}$ (i.p.) for 28 days and induced to ISO for 2 more days. Another group of rats were administered only PIC-I (10 mg/kg) for 28 days.

\section{Sample preparation}

All experimental rats were sacrificed (cervical dislocation) after 31 days by following $\mathrm{NIH}$ guidelines. Blood samples were immediately collected from the jugular vein, and serum samples were separated for further analysis. Cardiac tissues were excised immediately and a portion was fixed in $10 \%$ para-formaldehyde for histomorphological analysis. The remaining cardiac tissue was used for preparation of $10 \%$ homogenate (centrifuged at $8000 \times \mathrm{g}$ for $15 \mathrm{~min}$ at $4^{\circ} \mathrm{C}$ ), and the final supernatant was used. 


\section{Measurement of blood pressure (BP)}

Before rat sacrifice on the $31^{\text {st }}$ day, hemodynamic parameters like SAP (systolic arterial blood pressure), DAP (diastolic arterial blood pressure) and MAP (mean arterial total blood pressure) were noted with the help of CODA non-invasive tail-cuff BP monitor (Kent Scientific Corporation CT, USA).

\section{Assessment of cardiac infarct size/volume}

Cardiac infarct size/volume was evaluated according to the method of Kocak and others [22]. Transverse cardiac slices of thickness 2-3 $\mathrm{mm}$ were incubated in TTC stain (1\%) for $10 \mathrm{~min}$ at $37^{\circ} \mathrm{C}$, and fixed with $10 \%$ para-formaldehyde. If the cardiac tissue showed red color, it indicated non-infarct region (unaffected region). However, abnormal cardiac tissue showed pale grey color, which represented infarct region. A digital camera (Nikon, Eclipse50i, Japan) was used to picture the heart stained section and the infarct size/volume was calculated using Image Pro Plus software (V 6) from Media Cybernetics (MD, USA).

\section{Determination of cardiac lipid peroxidation products and enzymic antioxidants}

Lipid peroxidation product (malondialdehyde, MDA) and activities of enzymic antioxidants i.e. catalase (CAT) as well as superoxide dismutase (SOD) in heart tissue were measured using assay kit purchased from Nanjing Jian-cheng Bioenginering Inst (Nanjing, China), in line with the manufacturer's procedure.

\section{Evaluation of cardiac markers}

The concentration of serum cardiac markers like creatine kinase (CK-MB) and lactate dehydrogenase (LDH) were assayed by ELISA kit bought from Abcam (Cambridge, UK). Whereas, the level of cTnT was determined with kit purchased from Thermo Fisher Scientific (MA, USA).

\section{Determination of heart inflammatory markers}

Cytosolic and nuclear commercial fractionation kit (BioVision Inc., CA, USA) was employed for extracting nuclear and cytosolic fractions. Tumor necrosis factor alpha (TNF- $\alpha$ ), interleukin 6 (IL$6)$, interleukin 1 beta $(\mathrm{IL}-1 \beta)$ levels were quantified using ELISA kit purchased from $R$ \& $D$ systems (MN, USA). While, NF-kB p65 transcription factor assay kit (Abcam; Cambridge, UK) was used to check the levels of NF-KB p65 subunit in cardiac nuclear fraction of heart homogenate.

\section{Evaluation of cardiac apoptosis biomarkers}

The apoptotic bio-markers like caspase 3 and caspase9 were assayed in cardiac tissue homogenate using commercial ELISA kits from Beyotime, Biotechnlogy (Jiangsu, China) following supplier's protocol.

\section{Cardiac histomorphological analysis}

A slice of heart tissue fixed in $10 \%$ paraformaldehyde was blocked and tissue block were made using paraffin wax. The tissue blocks were sliced in to $5 \mathrm{~mm}$ using microtome. Then, the cardiac tissue slices were stained with $H$ \& $E$ staining solution and viewed under Olympus DP80microscope (Tokyo, Japan) and pictured using attached Olympus camera. Finally, the captured pictures were used to check for any histopathological using the help of Image Pro Plus software (V 6) from Media Cybernetics (MD, USA).

\section{Statistical analysis}

Values are denoted as the mean \pm standard error of mean (SEM). All the experimental groups were compared (multi-comparison) using one-way ANOVA and followed by Duncan's multi-range test. All statistical analyses were done with SPSS software version 21. Significant differences were set at $p<0.05$.

\section{RESULTS}

As shown in Figure 1, there were significant decreases $(p<0.01)$ in the levels of various hemodynamic parameters (BP)i.e. MAP, DAP and SAP in ISO-induced group, relative to the control group which was treated with only saline. Rat pre-treatment with PIC-1, followed by ISO treatment led to significant increases in the average levels of MAP, DAP and SAP, when compared with the ISO-injected rat groups.

Figure 2 shows the efficacy of PIC Ion cardiac infarct size/volume in control and isoproterenol induced heart damage (treated and untreated). An exponential increase $(p<0.01)$ in infarct size (large white region) was noted in ISOadministered rats, relative to control rats. Whereas PIC-I group rats pre-treated with only PIC-I showed marked decreases $(p<0.01)$ in infarct size (small white region), when compared with rats with ISO-induced heart damage. 


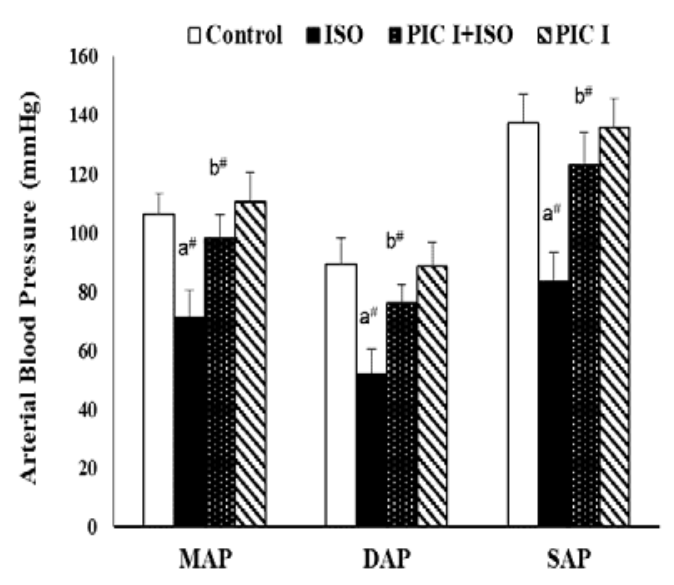

Figure 1: Effect of PIC-I on arterial blood pressure (tail). Values are denoted as mean \pm SEM. ${ }^{a \#} p<0.01$, ISO vs control; ${ }^{*} p<0.05$, PIC I+ISO vs ISO

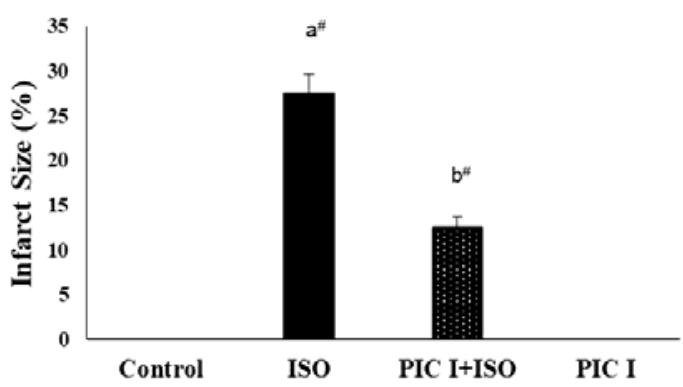

Figure 2: Effect of PIC I on cardiac infarct size (volume). Values are denoted as mean \pm SEM; ${ }^{a \# p}<$ 0.01 , ISO vs control; ${ }^{b^{*}} p<0.05$, PIC I+ISO vs ISO

Table 1 shows the efficacy of PIC-I on cardiac MDA and cardiac antioxidant enzymes. The levels of MDA were markedly increased $(p<$ 0.01 ) in ISO-treated rats than saline-treated rats. Nevertheless, administration of PIC-I for 28 days led to significant reduction $(p<0.01)$ in MDA to levels almost comparable with normal value. The activities of the antioxidant enzymes CAT and SOD were considerably attenuated $(p<0.01)$ in ISO-injected rats, but upon treatment with PIC-I, the activities of CAT and SOD were markedly increased $(p<0.01)$.

The efficacy of PIC-I on serum cardiac diagnostic marker enzymes were shown in Table 2. There was notable increase $(p<0.01)$ in the activities of cTnT, LDH, and CK-MB in ISO-induced MI rat model group. However, PIC-I pretreatment (10 $\mathrm{mg} / \mathrm{kg}$ ) prior to ISO exposure led to marked reductions in the activities of those cardiac markers enzymes as compared with Ml model (ISO) group.

Figure 3 shows the efficacy of PIC-I on cardiac inflammatory markers. The serum concentrations of NF-kB p65 subunit, IL-6, TNF- $\alpha, I L-1 \beta$ and were significantly raised $(p<0.01)$ in ISOinsulted rats as relative to control rats. However, treatment PIC-1 $(10 \mathrm{mg} / \mathrm{kg})$ for 28 days markedly reversed $(p<0.01)$ those inflammatory markers to near control levels.

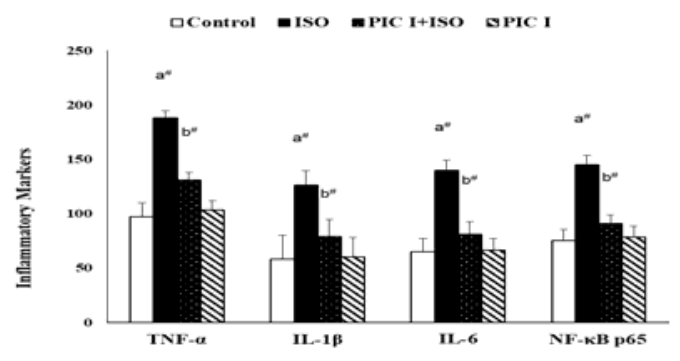

Figure 3: Effect of PIC-I on cardiac inflammatory markers. Values are presented as mean \pm SEM; ${ }^{a \#} p<$ 0.01 , ISO vs control; ${ }^{b *} p<0.05$, PIC I+ISO vs ISO (Unit: pg/mg protein)

Table 1: Efficacy of PIC-1 on cardiac MDA levels and antioxidants

\begin{tabular}{lcccc}
\hline Parameter & Control & ISO & PIC-I+ISO & PIC-I \\
\hline MDA (nmol/mg protein) & $0.62 \pm 0.08$ & $1.24 \pm 0.14^{\mathrm{a} \#}$ & $0.75 \pm 0.09^{\mathrm{b \#}}$ & $0.60 \pm 0.07$ \\
SOD (U/mg protein) & $4.95 \pm 0.40$ & $3.26 \pm 0.29^{\mathrm{a} \#}$ & $4.11 \pm 0.42^{\mathrm{b}^{*}}$ & $5.01 \pm 0.50$ \\
CAT (U/mg protein) & $16.36 \pm 1.50$ & $9.77 \pm 1.00^{\mathrm{a} \#}$ & $13.22 \pm 1.42^{\text {b\# }}$ & $15.98 \pm 1.45$ \\
\hline
\end{tabular}

Values are shown as mean \pm SEM; ${ }^{\text {\#\#}} p<0.01$, ISO vs control; ${ }^{b^{*}} p<0.05$, PIC-I+ISO vs ISO

Table 2: Effect of PIC I on cardiac diagnostic markers

\begin{tabular}{lcccc}
\hline Parameter & Control & ISO & PIC-I+ISO & PIC-I \\
\hline LDH (IU/L) & $90.05 \pm 7.40$ & $156.50 \pm 18.00^{\text {a\# }}$ & $107.23 \pm 13.70^{\text {b\# }}$ & $88.90 \pm 10.00$ \\
CK-MB (IU/L) & $68.72 \pm 7.20$ & $142.22 \pm 16.90^{\text {a\# }}$ & $88.20 \pm 9.30^{\text {b\# }}$ & $71.00 \pm 8.00$ \\
cTn T $(\mathrm{ng} / \mathrm{mL})$ & $0.55 \pm 0.07$ & $1.60 \pm 0.16^{\text {a\# }}$ & $0.79 \pm 0.08^{\text {b\# }}$ & $0.58 \pm 0.07$ \\
\hline Values are denoted as mean \pm SEM; ${ }^{\text {a\# }} p<0.01$, ISO vs control; ${ }^{\text {b }} p<0.05$, PIC I+ISO vs ISO. cTn T: cardiac \\
troponin T; ISO: isoproterenol; CP-MB: creatine phosphokinase isoform; PIC I: picroside I; LDH: lactate \\
dehydrogenase
\end{tabular}


The effect of PIC-I on cardiac apoptotic markers (caspase-3 and caspase-9) are shown in Figure 4 . There were significant increases $(p<0.01)$ in the mean value of apoptotic markers in ISOinduced rats, relative to saline-treated control rats. In contrast, the mean values of caspase- 3 and caspase-9 were notably reduced $(p<0.01)$ in PIC-I treated group, when compared with ISOinduced model rats.

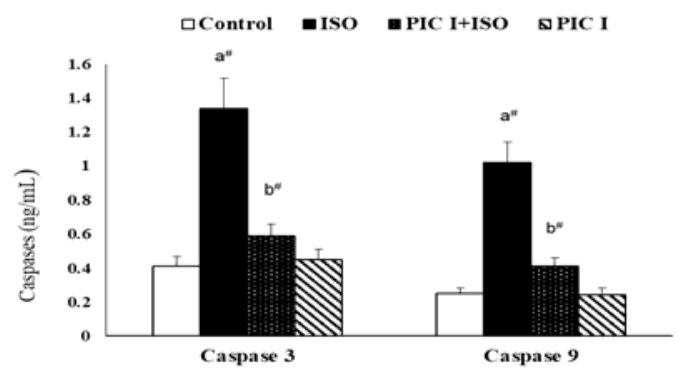

Figure 4: Effect of PIC-I on cardiac apoptotic markers (caspase-3 and caspase-9). Values are presented as mean \pm SEM; ${ }^{a \#} p<0.01$, ISO vs control; ${ }^{*} p<0.05$, $\mathrm{PIC}$ I+ISO vs ISO
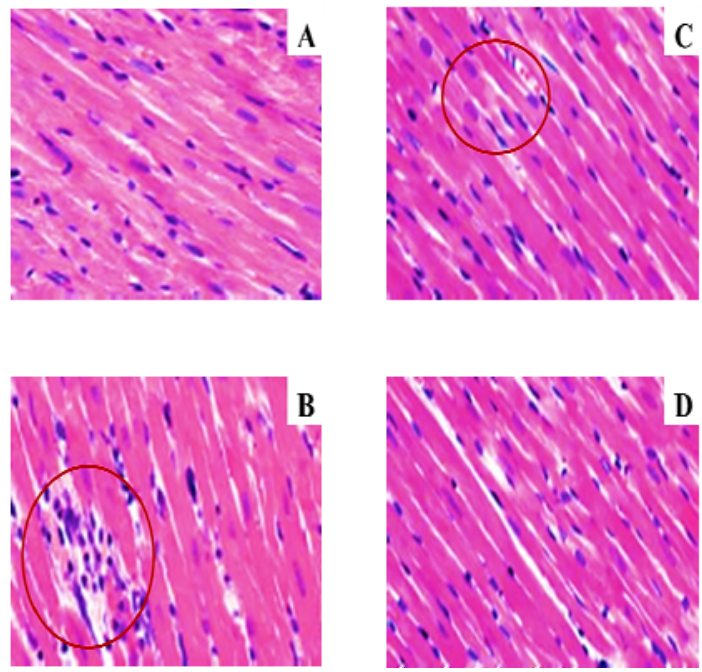

Figure 5: Effect of PIC-I on cardiac tissue morphological changes in control rats and rats with ISO-induced MI (treated and untreated), as shown using H\&E stain. Heart tissue slices of control rats showed normal cardiac architecture (A). Cardiac tissue slices of rats injected with ISO-1 showed considerable myofibrillar degeneration (circled), with increased leukocyte infiltration (inflammation) and subendocardial necrosis (B). In contrast, rats pre-treated with PIC-1 had lower degree of myofibrillar degeneration/disruption (circled) and decreased leukocyte infiltration (circled), with mild subendocardial necrosis (C), while heart tissue slices from $\mathrm{MI}$ rats given PIC-I showed normal cardiac architecture, similar to that of control rats (D). Scale bar: $100 \mu \mathrm{m}$
Figure 5 shows the efficacy of PIC-I on cardiac tissue morphological changes. Heart tissue slices of control rats showed normal cardiac architecture with clear myofibrillar profile. Similarly, the heart tissue slices of MI rats treated with PIC-I had normal cardiac architecture, similar that of control rats. The cardiac tissue of rats injected with ISO showed high degree of myofibrillar degeneration, with increased leukocyte infiltration (inflammation) and subendocardial necrosis. In contrast, rats pre-treated with PIC-1 had reduced myofibrillar degeneration/disruption and decreased leukocyte infiltration (circled), along with mild subendocardial necrosis.

\section{DISCUSSION}

This is the very first animal experiment conducted to study the protective efficacy of picroside 1 against ISO-exposed MI in rats. The outcome of the current study indicated that PIC-1 exerted cardioprotective effect through significant attenuation of infarct size, as well as levels of cardiac biomarkers, inflammatory markers and apoptotic markers. It also improved hemodynamic parameters, antioxidant status, and cardiac morphology. Previous studies showed that rats treated with ISO demonstrated reduced blood pressure owing to the reduced blood supply, which initiated reflex tachycardia (to compensate for the decreased blood pressure) and led to cardiac hypertrophy and remodeling $[20,23]$. Similarly, in the current study, ISO exposure also led to significant reductions in the hemodynamic parameters, i.e., MAP, DAP and SAP. However, rats pre-treated with PIC-I showed significant increases in values of MAP, DAP and SAP, thereby reversing the ISO-induced cardiac remodeling.

Cardiac infarct is the hallmark of $\mathrm{Ml}$, and it is usually detected via TTC staining process. The area of infarct size was large in rats injected with ISO owing to increased myocyte necrosis as a result of lack of blood supply and nutrients, as well as oxidative stress. However, pre-treatment of rats with PIC-I restored the antioxidant status and prevented free radical generation, thereby protecting the cardiac tissue from damage or injury. Oxidative stress (overproduction of free radicals which overpowers antioxidant capacity) and inflammation are the major factors involved in the etiology of Ml. Moreover, myocytes are greatly susceptible to free radical damage due to their low antioxidant enzyme contents, high oxygen requirement and high levels of polyunsaturated fatty acids[3,24].The degree of production of MDA (lipid peroxidation product)and the activities of antioxidant enzymes 
were significantly altered in rats given ISO due to increased oxidative stress. However, upon treatment with PIC-I for 28 days, there was significant restoration of the antioxidant enzyme activities and deceases in MDA production. This was due to the potent antioxidant effect of PIC-1. Studies have shown that PIC-1 extracted from Picrorhiza kurrooa can significantly scavenge free radicals owing to the presence of free hydroxyl groups which may contribute to anion scavenging property as well as anti-lipid peroxidation activity [14].

Cardiac diagnostic marker enzymes (cTn T, $\mathrm{LDH}$, and CK-MB) are used as indicators of cardiac dysfunction or damage especially during MI. The serum levels of cardiac markers were markedly elevated in ISO-induced $\mathrm{MI}$ rat model group. The ISO administration led to increased oxidative stress and inflammatory response owing to lack of sufficient amount of blood (nutrient and oxygen) which resulted insignificant disruption of myocyte cell integrity and permeability and results in discharge of cardiac marker from myocytes into extracellular fluid [6]. However, treatment with PIC-I (10 mg/kg) to ISO administered rats could led to significant reduction in the serum levels of those cardiac markers owing to the antioxidant and anti-lipid peroxidation properties of PIC-1 $[14,16]$.

It has been reported that high dose of ISO results in high levels of free radicals due to autooxidation of catecholamine, which in turn provokes inflammatory responses via upregulation of various pro-inflammatory cytokines via the NF- KB signaling pathway [25]. This is consistent with the significant increases in the values of numerous inflammatory markers (NF-kB p65 subunit, IL-6, TNF- $\alpha$ and IL-1 $\beta$ ) in ISO-induced $\mathrm{MI}$ animals. However, pre-treatment with PIC-I decreased concentrations of these inflammatory markers due to the potent antiinflammatory effect of PIC-1 $[13,15]$. Moreover, ISO administration markedly modulated the protein expressions of pro and anti-apoptotic proteins via caspase cascade especially caspase-3 and caspase-9 [26]. The results of this study also showed that caspase-3 and caspase9 were elevated in ISO-exposed rats, but those apoptotic markers were substantially reduced in rats pre-treated with PIC I, most likely due to the anti-inflammatory, anti-oxidant and anti-apoptotic properties of PIC-1 [14,15].

The cardiac tissue slices of rats administered ISO displayed considerable myofibrillar degeneration/disruption, increased leukocyte infiltration (inflammation) and sub-endocardial necrosis due to increased inflammatory response, oxidative stress and apoptosis/ necrosis. However, rats pre-treated with PIC-1 had less myofibrillar degeneration/disruption and decreased leukocyte infiltration, with mild subendocardial necrosis due to anti-apoptotic, antiinflammatory and antioxidant functions of PIC1[13-15]. There were no morphological changes in control or rats administered PIC-I alone, indicating that PIC-I did not produce any adverse effects. The major limitation of this study is that the possible involvement of other signaling pathways in the cardioprotective effect of PIC-I was not investigated.

\section{CONCLUSION}

Pre-treatment of rats with PIC I for 28 consecutive days prior to ISO administration results in strong cardioprotective effects via significant attenuation of infarct size, and levels of cardiac marker enzymes, apoptotic markers and inflammatory markers, with improved hemodynamic parameters, antioxidant status and cardiac morphology. Based on these results, PIC-I is a promising cardio-therapeutic agent against myocardial infarction. However, there is need for further studies on the mechanism behind its cardioprotective effect.

\section{DECLARATIONS}

\section{Acknowledgement}

This study was supported by Capital Medical University startup fund.

\section{Conflict of interest}

No conflict of interest is associated with this study

\section{Contribution of authors}

We declare that this work was done by the authors named in this article and all liabilities pertaining to claims relating to the content of this article will be borne by the authors. Yi Hao, Zhixiong Cui and Tong Liu designed and conducted this animal experiment. Yi Hao and Shuang Zhang helped in statistical analysis. Shuang Zhang and Tong Liu aided in drafting this manuscript. Yi Hao and Zhixiong Cui helped in histological analysis.

\section{Open Access}

This is an Open Access article that uses a funding model which does not charge readers or their institutions for access and distributed under the 
terms of the Creative Commons Attribution License (http://creativecommons.org/licenses/by/ 4.0) and the Budapest Open Access Initiative (http://www.budapestopenaccessinitiative.org/rea d), which permit unrestricted use, distribution, and reproduction in any medium, provided the original work is properly credited.

\section{REFERENCES}

1. Song S, Si LY. Klotho ameliorated isoproterenol-induced pathological changes in cardiomyocytes via the regulation of oxidative stress. Life Sci 2015; 135: 118123.

2. Liu J, Chen L, Lu, H. Cardioprotective effect of salvianolic acid $B$ against isoproterenol-induced inflammation and histological changes in a cardiotoxicity rat model. Trop $\mathrm{J}$ Pharm Res 2018; 17: 2187-2197.

3. Wong ZW, Thanikachalam PV, Ramamurthy S. Molecular understanding of the protective role of natural products on isoproterenol-induced myocardial infarction: $A$ review. Biomed Pharmacother 2017; 94: 1145-1166.

4. Peng D, Liu Q. Cardioprotective effect of Cycloalliin in Isoproterenol-induced myocardial infarction in albino rats: a mechanistic study. Arch Biol Sci 2016; 68: 789793.

5. Rajadurai $M$, Prince PS. Preventive effect of naringin on cardiac markers, electrocardiographic patterns and lysosomal hydrolases in normal and isoproterenolinduced myocardial infarction in Wistar rats. Toxicol 2007;230: 178-188.

6. Shukla SK, Sharma SB. $\beta$-Adrenoreceptor Agonist Isoproterenol Alters Oxidative Status, Inflammatory Signaling, Injury Markers and Apoptotic Cell Death in Myocardium of Rats. Ind J Clin Biochem 2015; 30: 2734.

7. Upaganlawar A, Gandhi H, Balaraman R. Isoproterenol induced myocardial infarction: Protective role of natural products. J Pharmacol Toxicol 2011; 6: 1-7.

8. Heusch G, Gersh BJ. The pathophysiology of acute myocardial infarction and strategies of protection beyond reperfusion: a continual challenge. Eur Heart J. 2016; 38:774-84.

9. Lecour S, T Lamont K. Natural polyphenols and cardioprotection. Mini-Rev Med Chem 2011; 11: 11911199.

10. Guo Y, Xu X, Li Q, Li Z, Du F. Anti-inflammation effects of picroside 2 in cerebral ischemic injury rats. Behav Brain Funct 2010; 6: 43-49.

11. Liu J, Liu BL, Zhang JQ, Zhang N. Hepatoprotective and choleretic action of Picrorhiza scrophulariae flora Pennell. Chin J New Drugs 2002; 11: 459-461.

12. Rathee D, Thanki M, Bhuva S, Anandjiwala S, Agrawal R. Iridoid glycosides-Kutkin, Picroside I, and Kutkoside from Picrorrhiza kurroaBenth inhibits the invasion and migration of MCF-7 breast cancer cells through the down regulation of matrix metalloproteinases: 1st Cancer Update. Arab J Chem 2013; 6(1): 49-58.
13. Singh $G B$, Bani $S$, Singh $S$, Khajuria A, Sharma $M L$, Gupta BD, Banerjee SK. Anti-inflammatory activity of the iridoids kutkin, picroside-1 and kutkoside from Picrorhizakurrooa. Phytother Res 1993; 7: 402-407.

14. Chander R, Kapoor NK, Dhawan BN. Picroliv, picroside-I and kutkoside from Picrorhiza kurrooa are scavengers of superoxide anions. BiochemPharmacol1992; 44: 180183.

15. Anand $P$, Kunnumakkara $A B$, Harikumar KB, Ahn KS, Badmaev V, Aggarwal BB. Modification of cysteine residue in $p 65$ subunit of nuclear factor- $k B$ (NF-KB) by Picroliv suppresses NF-KB-regulated gene products and potentiates apoptosis. Cancer Res 2008; 68: 88618870.

16. Zhang S, Lu Y, Zheng J, Qin H, Qiu X, Huang H, Mo G, Liang $S$, Zhang $Y$. Picroside I inhibits asthma phenotypes by regulating Tbet/GATA-3 ratio and Th1/Th2 balance in a murine model of asthma. Trop $J$ Pharm Res 2018; 17: 1777-1782.

17. Li P, Matsunaga K, Yamakuni T, Ohizumi Y. Potentiation of nerve growth factor-action by picrosides I and II, natural iridoids, in PC12D cells. Eur J Pharmacol. 2000; 406: 203-208.

18. Dwivedi Y, Rastogi R, Garg NK, Dhawan BN. Picroliv and its components kutkoside and picroside I protect liver against galactosamine-induced damage in rats. Pharmacol Toxicol 1992; 71: 383-387.

19. Kumar SH, Anandan R, Devaki T, Kumar MS. Cardioprotective effects of Picrorrhiza kurroa against isoproterenol-induced myocardial stress in rats. Fitoterapia 2001; 72:402-405.

20. Nandave M, Ojha SK, Kumari S, Nag TC, Mehra R, Narang R, Arya DS. Cardioprotective effect of root extract of Picrorhiza kurroa (Royle Ex Benth) against isoproterenol-induced cardiotoxicity in rats. Indian J Exp Biol 2013; 51: 674-701.

21. Li JZ, Xie MQ, Mo D, Zhao XF, Yu SY, Liu LJ, Wu C, Yang Y. Picroside II protects myocardium from ischemia/reperfusion induced injury through inhibition of the inflammatory response. Exp Therapeut Med 2016; 12: 3507-3514.

22. Kocak C, Kocak FE, Akcilar R, Isiklar OO, Kocak H, Bayat Z, Simsek H, Taser F, Altuntas I. Molecular and biochemical evidence on the protective effects of embelin and carnosic acid in isoproterenol-induced acute myocardial injury in rats. Life Sci. 2016; 147: 1523.

23. Xu L, Deng Y, Feng L, Li D, Chen X, Ma C, Liu X, Yin J, Yang $M$, Teng F, Wu W. Cardio-protection of salvianolic acid $B$ through inhibition of apoptosis network. PloS One 2011; 6: e24036.

24. Neri M, Fineschi V, Di Paolo M, Pomara C, Riezzo I, Turillazzi E, Cerretani D. Cardiac oxidative stress and inflammatory cytokines response after myocardial infarction. Curr Vasc Pharmacol 2015; 13: 26-36.

25. Sun SJ, Wu XP, Song HL, Li GQ. Baicalin ameliorates isoproterenol-induced acute myocardial infarction through iNOS, inflammation, oxidative stress and

Trop J Pharm Res, April 2020; 19(4):787 
Hao et al

P38MAPK pathway in rat. Int J Clin Exp Med 2015; 8: 22063.

26. Othman Al, Elkomy MM, El-Missiry MA, Dardor $M$ Epigallocatechin-3-gallate prevents cardiac apoptosis by modulating the intrinsic apoptotic pathway in isoproterenol-induced myocardial infarction. Eur $J$ Pharmacol 2017; 794: 27-36. 